\title{
BMJ Open Symptom burden in multimorbidity: a population-based combined questionnaire and registry study from Denmark
}

\author{
Tora Grauers Willadsen (D) , ${ }^{1}$ Volkert Siersma, ${ }^{1}$ Dagny Ros Nicolaisdottir, ${ }^{2}$ \\ Dorte Jarbol (1) , ${ }^{3}$ Ann Dorrit Guassora, ${ }^{1}$ Susanne Reventlow (i) , \\ Rasmus Køster-Rasmussen ${ }^{1}$
}

To cite: Willadsen TG, Siersma V, Nicolaisdottir DR, et al. Symptom burden in multimorbidity: a population-based combined questionnaire and registry study from Denmark. BMJ Open 2021;11:e041877. doi:10.1136/ bmjopen-2020-041877

- Prepublication history and supplemental material for this paper is available online. To view these files, please visit the journal online (http://dx.doi. org/10.1136/bmjopen-2020041877).

Received 22 June 2020 Revised 16 February 2021 Accepted 18 March 2021

Check for updates

(c) Author(s) (or their employer(s)) 2021. Re-use permitted under CC BY-NC. No commercial re-use. See rights and permissions. Published by BMJ.

${ }^{1}$ Department of Public Health, University of Copenhagen, Copenhagen, Denmark

${ }^{2}$ The Research Unit for General Practice, University of Copenhagen, Copenhagen, Denmark

${ }^{3}$ The Research Unit for General Practice, University of Southern Denmark, Odense, Denmark

Correspondence to

Dr Tora Grauers Willadsen;

tora.willadsen@sund.ku.dk

\section{ABSTRACT}

Objective Patients with multimorbidity may carry a large symptom burden. Symptoms are often what drive patients to seek healthcare and they also assist doctors with diagnosis. We examined whether symptom burden is additive in people with multimorbidity compared with people with a single morbidity.

Design This is a longitudinal cohort study drawing on questionnaire and Danish national registry data. Multimorbidity was defined as having diagnoses from at least two out of ten morbidity groups. Associations between morbidity groups and symptom burden were estimated with multivariable models.

Participants In 2012, 47452 participants from the Danish Symptom Cohort answered a questionnaire about symptoms (36 symptoms in total), including whether symptoms were affecting their daily activities (impairment score) and their worries about present symptoms (worry score) (the highest score among the 36 symptoms on a 0-4 scale).

Main outcome measure The primary outcome was symptom burden.

Results Participants without morbidity reported 4.77 symptoms (out of 36 possible). Participants with one, two or three morbidities reported more symptoms than patients without morbidity ( 0.95 (Cl 0.86 to 1.03 ), 1.87 (Cl 1.73 to 2.01 ) and 2.89 ( $\mathrm{Cl} 2.66$ to 3.12), respectively). Furthermore, they reported a higher impairment score $(0.36$ ( 0.32 to $0.39), 0.65$ (0.60 to 0.70 ) and 1.06 (0.98 to 1.14)) and a higher worry score ( 0.34 (0.31 to 0.37 ), 0.62 (0.57 to 0.66 ) and $1.02(0.94$ to 1.10$))$ than participants without morbidity. In 45 possible combinations of multimorbidity (participants with two morbidities), interaction effects were additive in 37,41 and 36 combinations for the number of symptoms, impairment score and worry score, respectively. Conclusion Participants without morbidity reported a substantial number of symptoms. Having a single morbidity or multimorbidity resulted in approximately one extra symptom for each extra morbidity. In most combinations of multimorbidity, symptom burden was additive.

\section{INTRODUCTION}

Multimorbidity, most often defined as the co-occurrence of two or more chronic

\section{Strengths and limitations of this study}

- This study combines high-quality data from Danish national registries and questionnaire data from the background population.

- The questionnaire used in this study draws on a breadth of symptoms indicative of both serious and less harmful diseases.

- The selection of symptoms in the questionnaire is not exhaustive, which may induce artificial differences between morbidities; nevertheless, this does not bias synergy estimates.

- Multimorbidity is defined as having two or more morbidities; in this study, a morbidity is defined as having one or more chronic diagnoses from one of ten predefined morbidity groups: lung, musculoskeletal, endocrine, mental health, cancer, neurological, gastrointestinal, cardiovascular, genitourinary and sensory.

diseases, ${ }^{1}$ affects mental health, ${ }^{2}$ quality of life $^{3}$ and survival, ${ }^{4}$ and patients with multimorbidity often struggle to manage their symptoms. ${ }^{5}$ Symptoms play a major part in how people self-rate their health. ${ }^{6}$ Factors like disability, duration and feelings of vulnerability are important drivers for how a sensation gradually turns into a symptom, ${ }^{7}$ where interpretations of danger and intensity can be decisive in making a contact with healthcare. ${ }^{8}$ Hence, the presence of symptoms is important for doctors to be able to formulate a diagnosis, and thereby symptoms become a pathway through which a person becomes a patient. ${ }^{9}$ Symptoms, however, can be difficult to explain and doctors may focus solely on relieving them. ${ }^{9}{ }^{10}$ Additionally, diagnoses are not always helpful in explaining symptom experiences, ${ }^{11}$ and having one diagnosis increases the risk of being diagnosed with other diseases. ${ }^{12}$ Patients with multimorbidity 


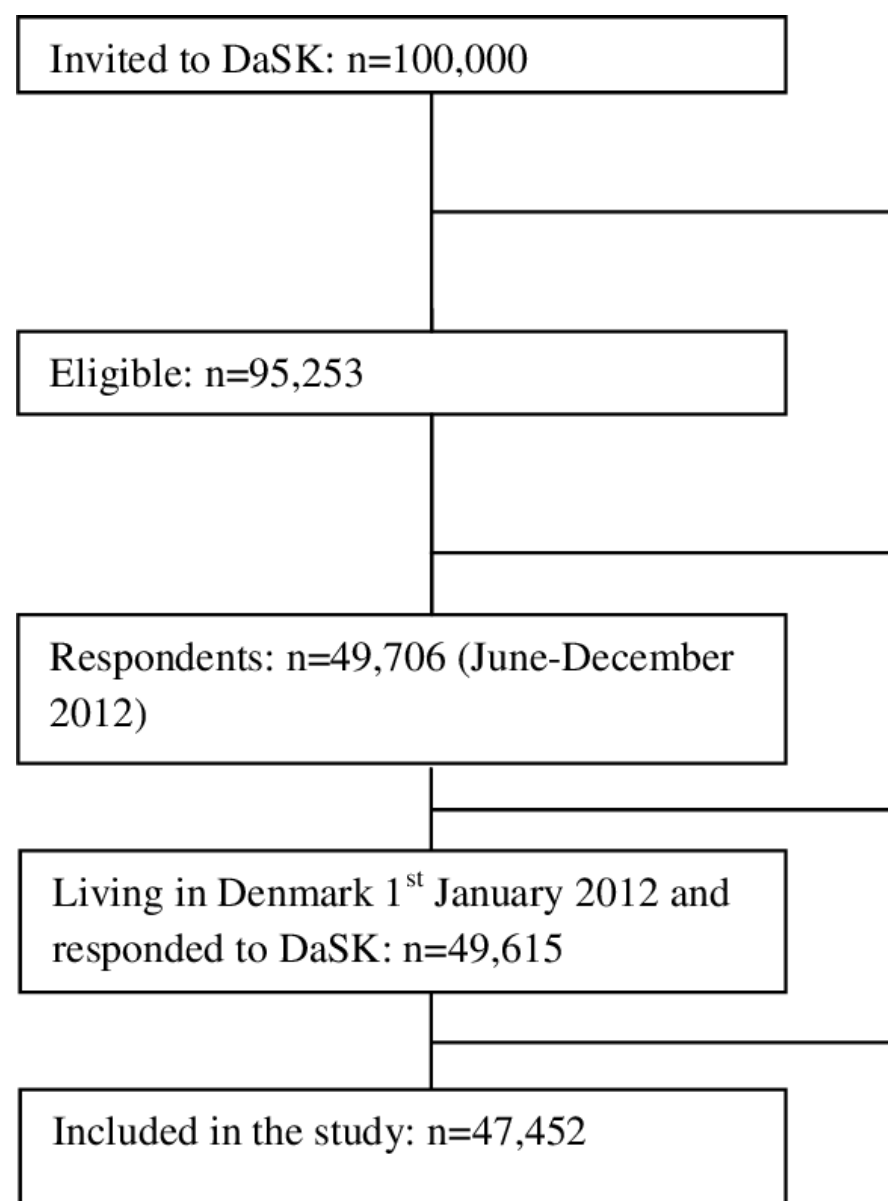

Figure 1 Study population. DaSK, Danish Symptom Cohort.

report a considerable symptom burden, ${ }^{513}$ and more focus on symptom management in multimorbidity has been suggested. ${ }^{14}$ Therefore, an understanding of the relationship between diagnoses and symptom burden is warranted, and especially an understanding of how symptom burden is experienced when diagnoses occur in multiples, that is, multimorbidity. The aim of this study was to explore symptom burden in patients with one of ten morbidities compared with symptom burden in patients with multimorbidity. We hypothesised that symptom burden in multimorbidity was additive, that is, symptoms in patients with multimorbidity were equivalent to the sum of the symptoms attributable to the single morbidities.

\section{METHODS}

\section{Study design and population}

The study was a longitudinal cohort study. Participants were from the Danish Symptom Cohort, a populationbased study conducted in Denmark in June-December 2012. People invited to participate in the study were living in Denmark on 1 January 2012 (baseline). Of 100000 adults ( $\geq 20$ years) randomly selected from the general Danish population, 95253 were eligible and invited to participate (figure 1). Of these, 49706 (52.2\%)
Excluded: $\mathrm{n}=4747$

- Severe illness (including dementia): 1972

- Death before start: 315

- Languages problems: 885

- Emigration: 614

- Unknown address: 961

Non-respondents: $\mathrm{n}=45,547$

Did not want to participate: 25,690

No contact with the person: 19,539

Indicated other reason for not participating: 318

Disappeared: $\mathrm{n}=91$

Neither in the Civil Registration System nor in the Register of Causes of Death

Excluded: $n=2163$

Not lived continuously in Denmark ten years

prior to baseline

$\left(1^{\text {st }}\right.$ January 2002-31 ${ }^{\text {st }}$ December 2011) completed the study questionnaire (online supplemental file 1). ${ }^{15}$

All Danish-born and immigrant populations in Denmark have a unique personal identification number in the Danish Civil Registration System. ${ }^{16}$ The register contains information about age, sex, vital status and so on, and enables information from different Danish registries to be linked. Information on diagnoses leading to either inpatient or outpatient care in the hospital sector was collected from the Danish National Patient Register, ${ }^{17}$ the Danish Cancer Registry ${ }^{18}$ and the Danish Psychiatric Central Research Register. ${ }^{19}$ Thus, only diagnoses from secondary care were included in the study. Information on education, ${ }^{20}$ work status, ${ }^{21}$ family income, ${ }^{22}$ assets (banks, stocks, bonds and housing), ${ }^{22}$ degree of urbanisation and cohabitation status was obtained from the nationwide registries at baseline.

\section{Symptom data}

The Danish Symptom Cohort was established to investigate symptom experience in the general population and healthcare-seeking in relation to general practice. According to the random sample selected from the Danish population, the cohort included both healthy people and people with diseases. Several articles have been published with data from the cohort. ${ }^{23-25}$ The focus 
of the present study was symptom burden in people with multimorbidity and variables for the study were selected accordingly. ${ }^{23}$ The median age of the participants in the Danish Symptom Cohort was 52 years (IQR 40-64) and for non-participants it was 50 years (IQR 36-67). The respondents were reasonably representative of the study sample, but non-respondents were more often men, unmarried, with lower education, lower income level and with a generally looser attachment to the labour market.

The survey consisted of a web-based questionnaire supported by a telephone interviewer if warranted. The questionnaire was electronic and designed so that it was not possible to skip items, and therefore there were no missing values for those who completed it. The process of developing the questionnaire has been described by Rasmussen et al. ${ }^{15}$ The questionnaire had five domains: three about experience of symptoms and how participants acted on them, and two about factors related to symptom experience and healthcare-seeking behaviour (online supplemental file 1). The questionnaire included 38 general symptoms, as well as 2 specific symptoms for men and 4 for women (44 symptoms in total). The first sentence in the questionnaire was 'We are interested to hear if you have experienced any bodily sensations, symptoms, or discomfort within the last fourweeks'. For the general symptoms the following phrase was used: 'Have you experienced any of these within the last 4 weeks?' Respondents had the opportunity to tick more than one box in a list presenting the 38 general symptoms (online supplemental file 1). Eight symptoms were excluded in the present analyses as six of them were gender-specific (as mentioned above) and would have made comparisons between men and women difficult, and two symptoms 'coughing up blood' and 'blood in vomit' had low prevalence in the data. Therefore, we included 36 symptoms in the analyses (online supplemental file 2).

The questionnaire also included questions about how each symptom affected usual daily activities (impairment score) and the participant's concern about each symptom (worry score): 'Within the last 4 weeks, to what extent did you experience that the following symptoms or discomfort interfered with your usual daily activities?' and 'Within the last 4 weeks, to what extent were you concerned about the following symptoms or discomfort?' For each symptom, there were five response categories: 'not at all', 'slightly', 'moderate', 'quite a bit' and 'extremely'. The response categories were transformed into a numeric scale $(0-4)$, where the experience rated 4 is worse than the experience rated 1.

\section{Symptom burden}

Three components defined symptom burden: (1) number of symptoms (number score 0-36); (2) how symptoms affect usual daily activities (impairment score $0-4$ per symptom reported; and (3) concern about the symptoms (worry score $0-4$ per symptom reported).

For the impairment score and the worry score, we included the symptom with the highest score in the analyses; for example, if a patient scores 3 for tiredness and scores 1 for dizziness, the overall score is 3 . This is because we anticipated that the most burdensome symptoms affect quality of life more heavily than a number of minor symptoms. ${ }^{26}$

\section{Multimorbidity}

Information on diagnoses was retrieved from the nationwide health registries in the 10 -year period preceding baseline (1 January 2002-31 December 2011). Participants were excluded if they had not been living continuously in Denmark during this 10 -year period. Chronic disease diagnoses were grouped into 10 domains: lung, musculoskeletal, endocrine, mental health, cancer, neurological, gastrointestinal, cardiovascular, genitourinary and sensory. In each domain, relevant diagnoses from the International Classification of Diseases, 10th edition were included (online supplemental file 3 ). Morbidity is defined as having a diagnosis from one domain, and multimorbidity is defined as having a diagnosis from two or more different domains. ${ }^{27}$ This definition rests on the assumption that it is more complex and realistic from a physiological and organisational point of view if the patient is living with diagnoses from different domains of the body. ${ }^{27}$ Often two chronic diagnoses refer to the same disease entity, for example, myocardial infarction and congestive heart failure. Therefore, our definition of multimorbidity relates more closely to how healthcare is organised and it grasps some aspects of complexity to a greater extent than counting individual diagnoses (online supplemental file 3). People who do not have any of the diagnoses included in the 10 domains were considered to have no morbidity.

\section{Statistical analysis}

Symptom burden in people with a single morbidity and multimorbidity was explored using multivariate analyses adjusted for age, sex, socioeconomic status (highest completed education, income, assets and work status), degree of urbanisation, cohabitation status, smoking and alcohol consumption. Excess symptom burden for people with multimorbidity (combinations of two diagnosis domains) was assessed in multivariable linear regression models. For each of the three measures of symptom burden (number of symptoms, impairment score and worry score), the $(10 \times 9) / 2=45$ regression coefficients pertaining to the two-way interactions between diagnosis domains were retained from a multivariable linear regression on all combinations of diagnosis domains, adjusted for the same covariates as mentioned above. These coefficients were directly interpreted as the synergy effect, that is, excess symptom burden associated with having diagnoses from two diagnosis domains relative to the sum of the symptom burden associated with having a diagnosis from the diagnosis domains individually. Analyses were performed using SAS V.9.4.

\section{Patient and public involvement}

No patients nor any other members of the public were involved in the design, conduct or reporting of this study.

Informed consent was obtained from all participants. 


\section{RESULTS}

The study sample consisted of 47452 people $\geq 20$ years old (figure 1), out of whom 17227 (36.3\%) had a single morbidity and $5652(11.9 \%)$ had multimorbidity (table 1$)$. Overall, $43228(91.1 \%)$ reported symptoms (table 1 and online supplemental file 4 ), with $92.8 \%$ of people with single morbidity or multimorbidity reporting at least one symptom and $90.2 \%$ of people without morbidity reporting at least one symptom. On average, the participants experienced 4.8-7.4 symptoms depending on morbidities within the last 4 weeks (online supplemental table A).

\section{Morbidity and symptom burden}

Participants without morbidity reported 4.77 symptoms (SD 3.92) on average (online supplemental table A). The more morbidities a participant had, the more symptoms they reported. In multivariable analysis adjusted for confounders, each extra morbidity was associated with approximately one extra symptom (table 2).

Participants without morbidity reported a mean impairment score of 2.74 (SD 1.46). The more morbidities a participant had, the higher their reported impairment score (table 2). In multivariable analysis adjusted for confounders, each extra morbidity was associated with an approximately 0.35 higher impairment score (participants with one, two or three morbidities) than participants without morbidity. However, in participants with four or more morbidities, there was a levelling off in added impairment score with the number of morbidities (table 2).

Participants without morbidity reported a worry score of 2.16 (SD 1.38). The more morbidities, the higher the worry score participants reported (table 2). In multivariable analysis adjusted for confounders, each extra morbidity was associated with an approximately 0.34 higher worry score (participants with one, two or three morbidities) than participants without morbidity. In participants with four or more morbidities, however, there was a levelling off in added worry score for each additional morbidity (table 2 ).

\section{Multimorbidity and interaction effect of symptom burden}

Participants with multimorbidity (confined to participants with two morbidities) were analysed for interaction effects regarding their number of symptoms, impairment score and worry score. We did this to estimate if the symptom burden was additive (equivalent to the sum of two individual diagnoses), infra-additive (less than) or supra-additive (greater than) in specific combinations of multimorbidity.

The number of symptoms reported by participants with specific combinations of multimorbidity is outlined in online supplemental tables A and B and depicted in figure 2. In multivariable analyses adjusted for confounders, the number of symptoms reported by the participants was additive in 37 out of 45 combinations of multimorbidity. The combinations lung-mental health, cancer-heart and cancer-sensory were associated with a supra-additive number of symptoms (online supplemental table $\mathrm{C}$ ). On the other hand, the combinations lung-musculoskeletal, endocrine-mental health, neurological-mental health, cancer-kidney and heart-kidney were associated with an infra-additive number of reported symptoms (online supplemental table $\mathrm{C}$ ).

Impairment scores as reported by participants with multimorbidity are outlined in online supplemental tables $\mathrm{D}$ and $\mathrm{E}$ and depicted in figure 3. In multivariable analyses adjusted for confounders, the impairment score was additive in 41 out of 45 combinations of two morbidities. The combination cancer-sensory was associated with a supra-additive impairment score. The combinations lung-gastrointestinal, cancer-kidney and heart-kidney were associated with an infra-additive impairment score.

Worry scores as reported by participants with multimorbidity are outlined in online supplemental tables $\mathrm{F}$ and $\mathrm{G}$ and depicted in figure 4. In multivariable analyses the worry score was additive in 36 out of 45 combinations of two morbidities. The combinations cancer-mental health, cancer-neurological and cancer-sensory were associated with a supra-additive worry score. The combinations lung-musculoskeletal, lung-kidney, endocrine-kidney, cancer-kidney, neurological-kidney and heart-kidney were associated with an infra-additive impairment score.

\section{DISCUSSION \\ Main findings}

Participants without morbidity reported a number of symptom experiences (mean 4.8, median 4), and one extra symptom was added with each additional morbidity. Patients with a single morbidity, therefore, reported 5.8 symptoms, patients with three morbidities reported 7.8 symptoms, and so forth. Thus, the number of symptoms was additive with one extra symptom for each extra morbidity.

The same pattern was seen for how the symptoms interfered with the participants' daily activities and how much the participants were concerned about their symptoms. An impairment score (2.7) and a worry score (2.2) were reported by participants with no morbidity. Each morbidity added approximately 0.3 to the impairment and worry scores (up to three morbidities). Hereafter the impairment and worry scores levelled off to a slow increase with the number of morbidities (four, five and six). Thus, the scores for how symptoms interfered with the participants' daily activities and how much the participants were concerned about their symptoms were relatively high in participants without morbidity, and the increase in impairment and worry scores was additive for every extra morbidity up to three. Hereafter, each morbidity (from four to seven morbidities) did not add much to the impairment and worry scores.

In regard to symptom burden among specific combinations of multimorbidity, most combinations followed an additive pattern, that is, the reported extra symptom 


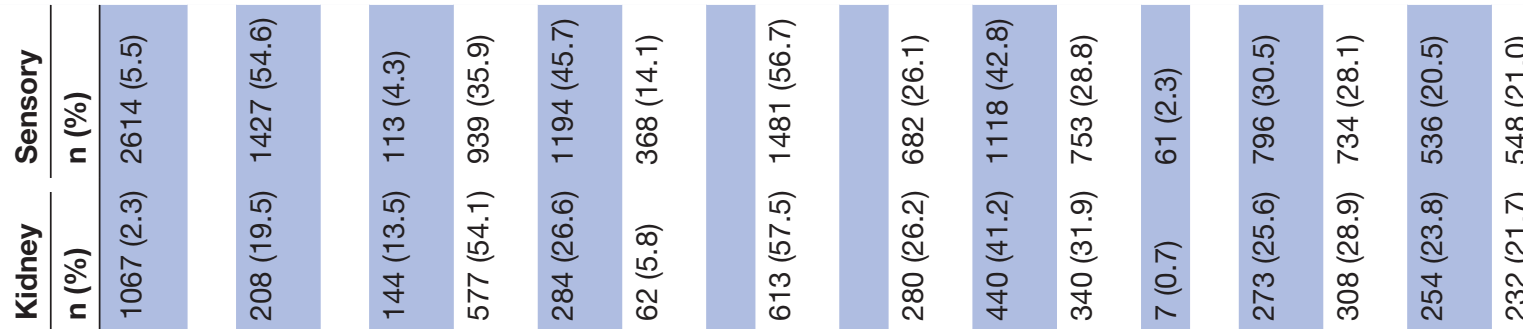

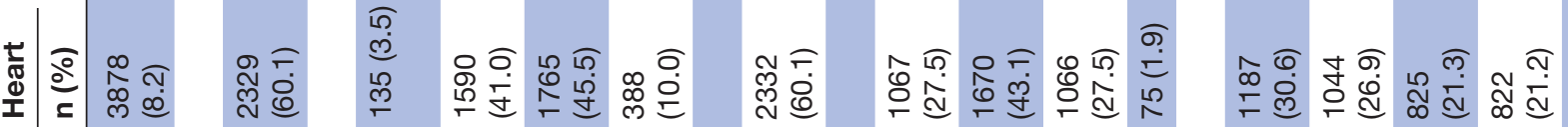

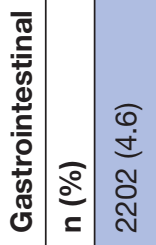

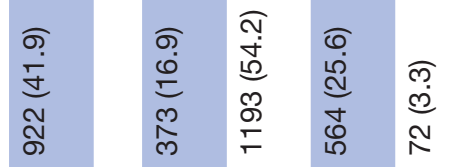

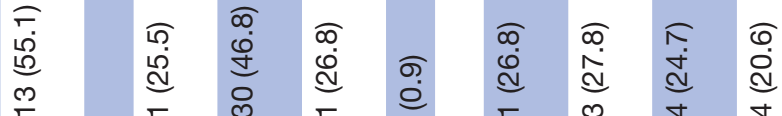

는

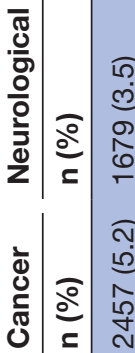

อิ

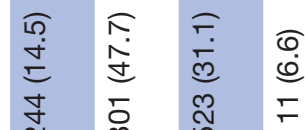

ช $\sigma$ o

कृ

बे दु

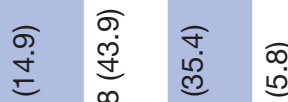

$\hat{0}=\begin{array}{cccc}0 & 0 & 0 & 0\end{array}$

幖

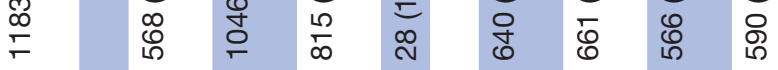

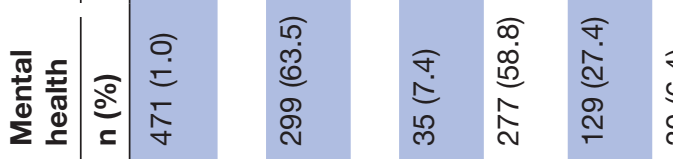

ब

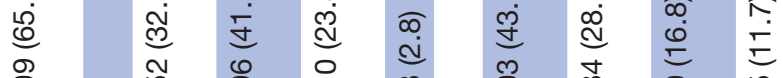

वे त

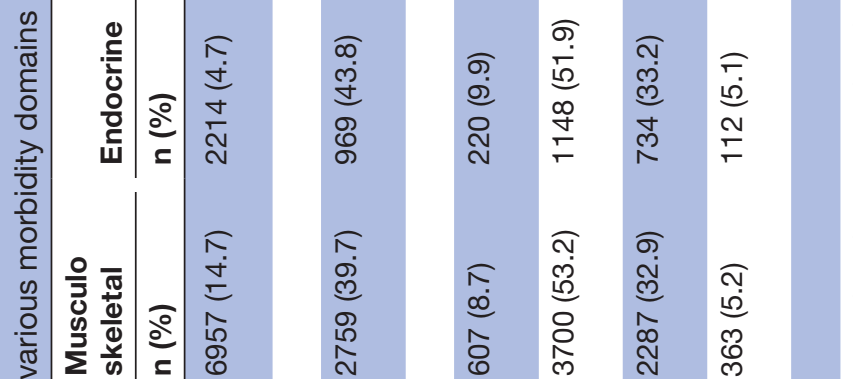

ฮิ

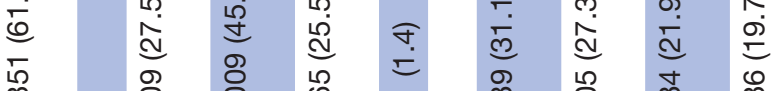

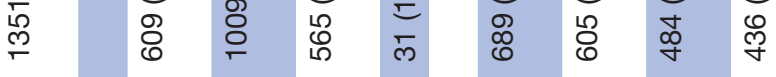

ब

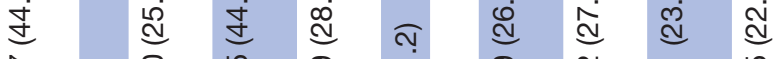

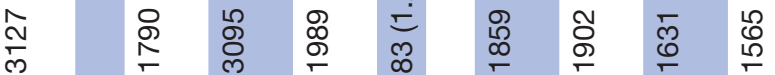

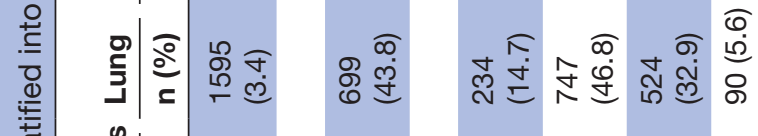

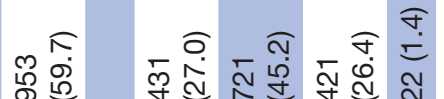

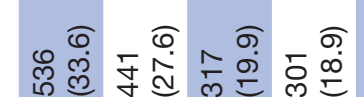

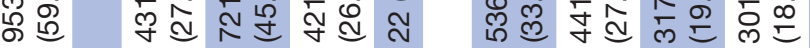

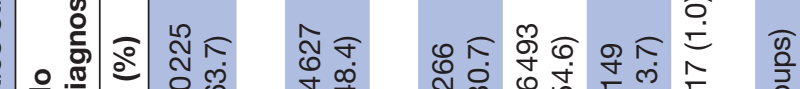

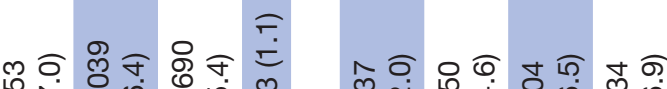

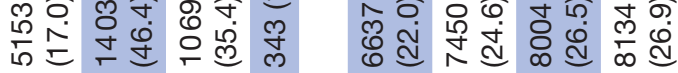




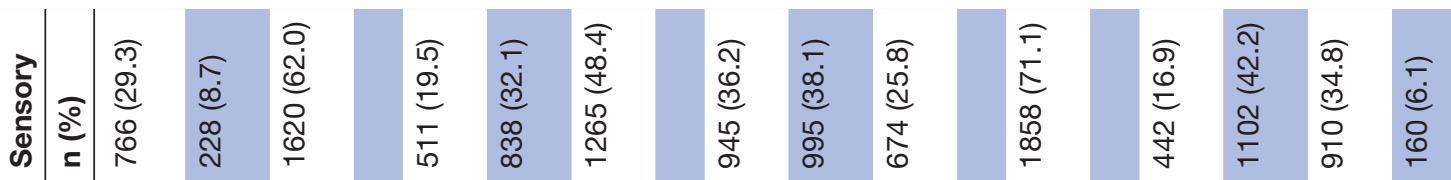

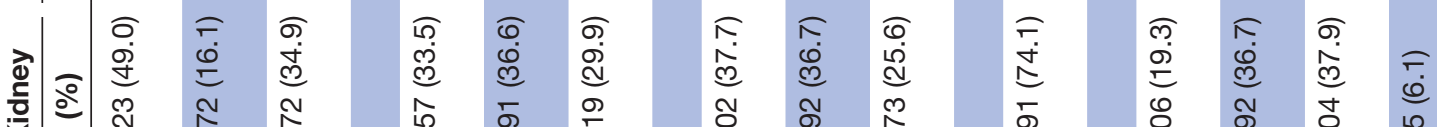

守

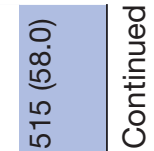

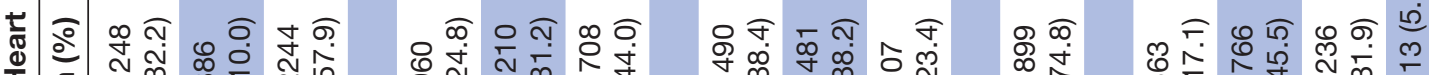

유다.

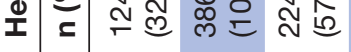

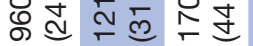

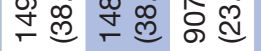

$\stackrel{N}{N}$

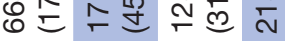

Nิ

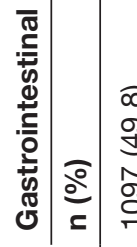

o

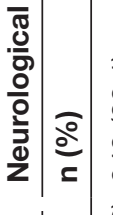

仓.

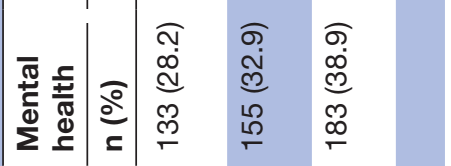

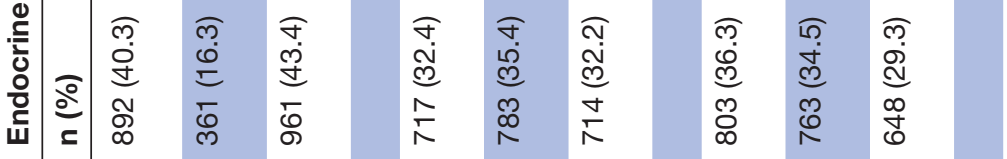

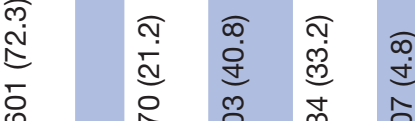

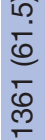

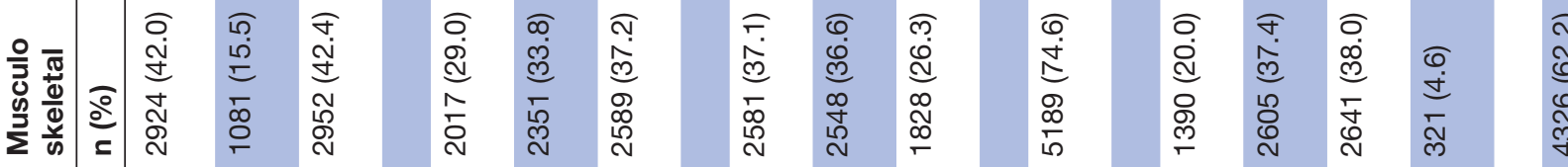

สุ

ฮิ

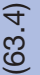

$\stackrel{\odot}{\mathscr{\infty}}$

当 $\frac{\omega}{\omega}$

兽 2 要|

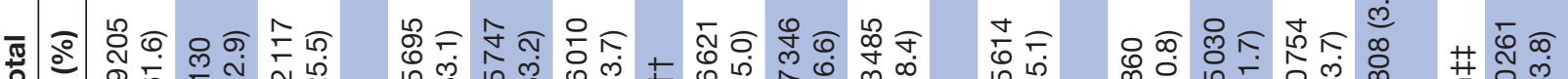
苞 占点 次 占 


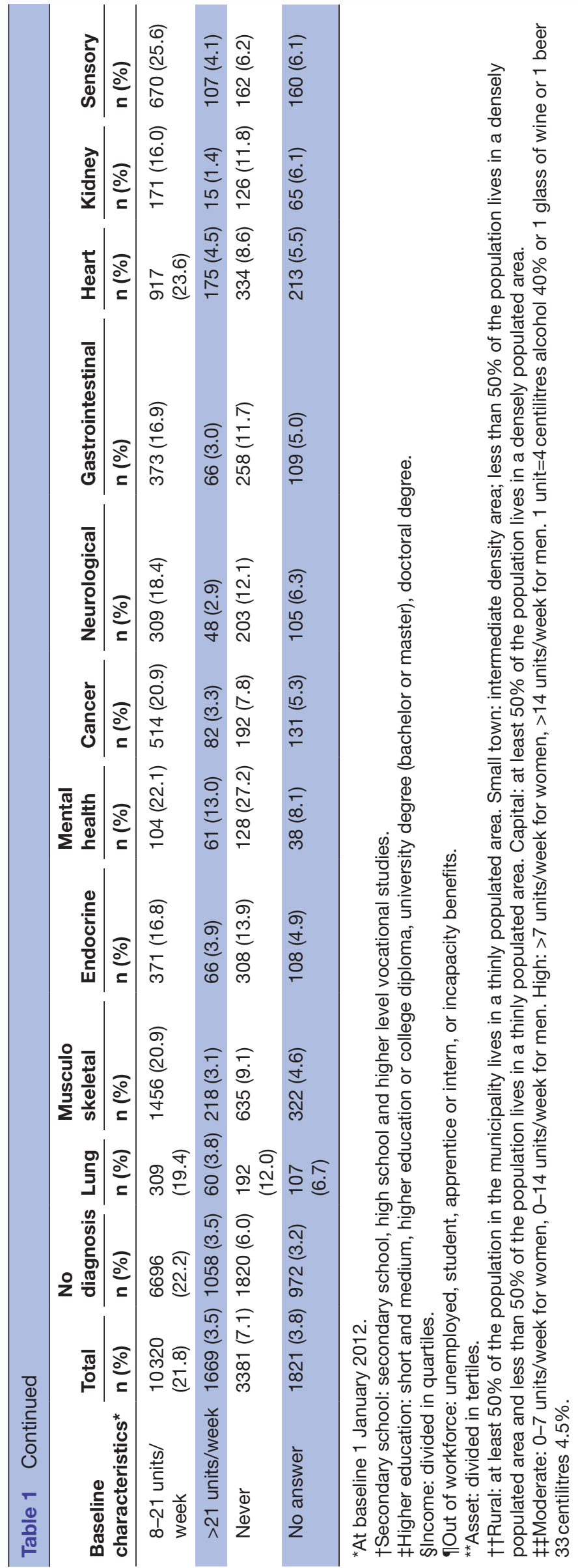

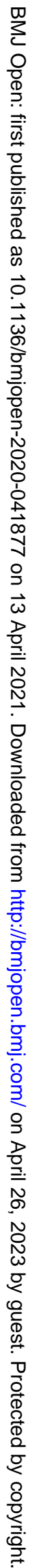




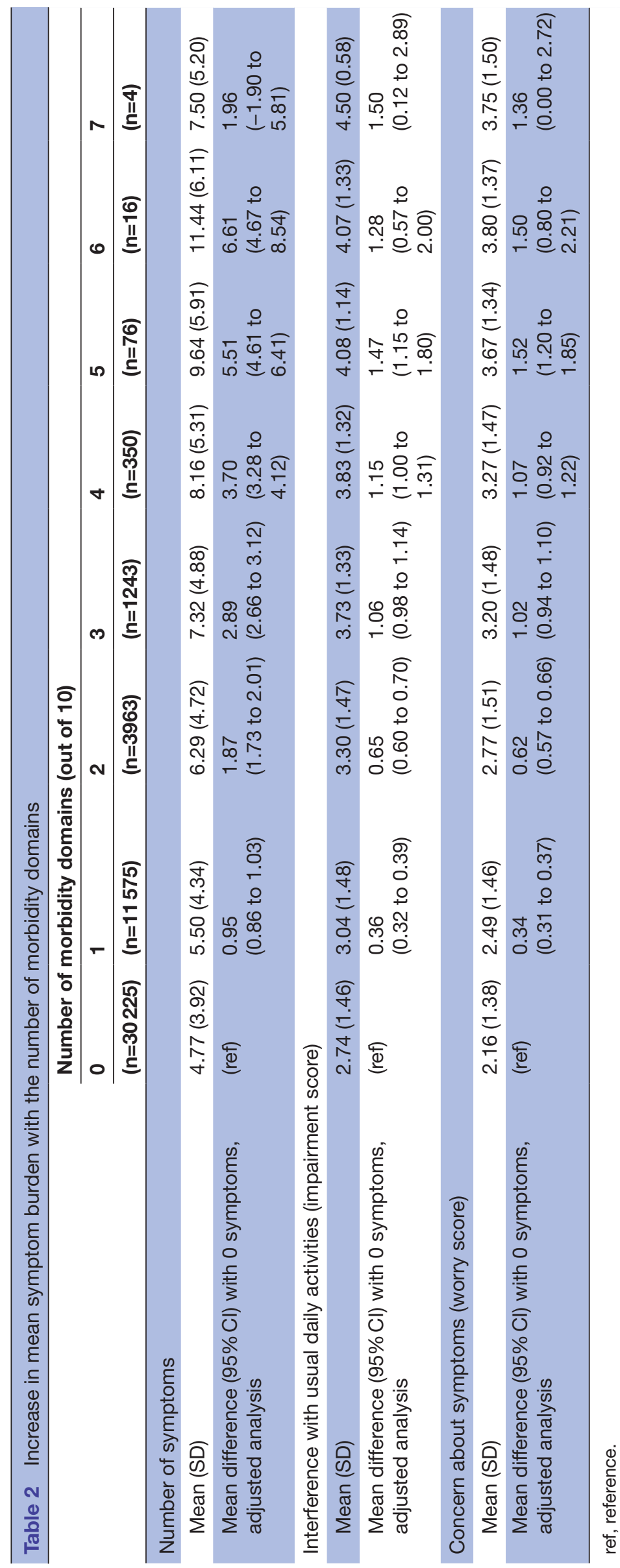

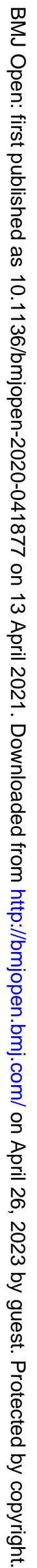




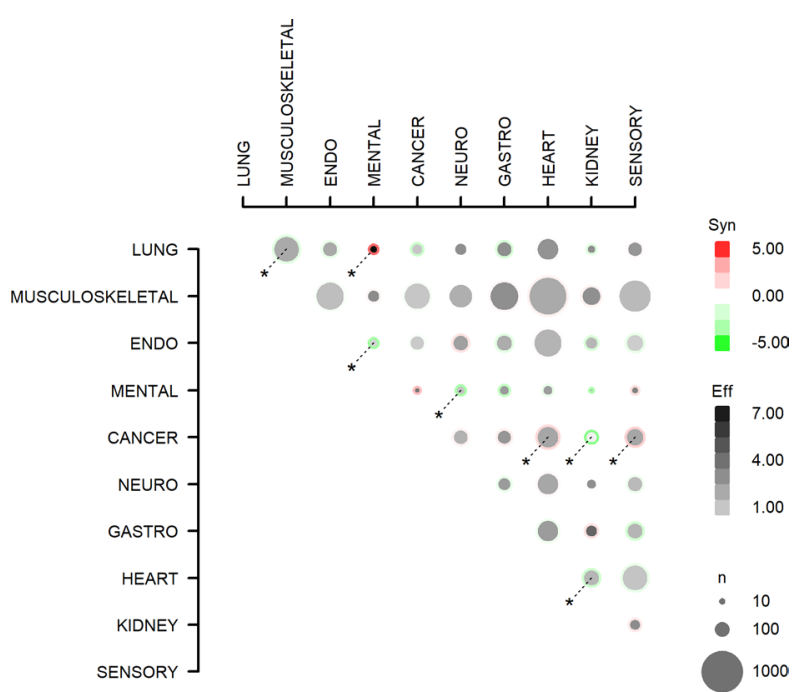

Figure 2 All (45) combinations of multimorbidity (two diagnosis groups) and the association with number of symptoms. Effects with $p<0.05$ are marked with *. Eff, effect: the number of symptoms for the multimorbidity combination. Syn, synergy: the excess number of symptoms for multimorbidity, compared with the sum of symptoms from two people having the two morbidity domains individually. $n$ is the number of people with the multimorbidity combination. ENDO, endocrine; GASTRO, gastrointestinal; NEURO, neurological.

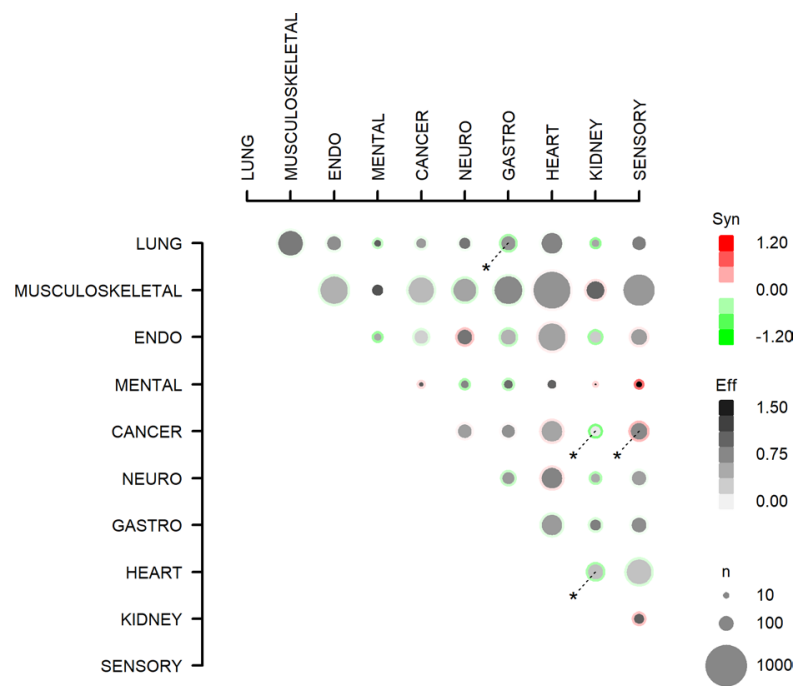

Figure 3 All (45) combinations of multimorbidity (two diagnosis groups) and the association with influence on daily activities. Effects with $\mathrm{p}<0.05$ are marked with * Eff, effect: the interference score for the multimorbidity combination; Syn, synergy: the excess interference with usual daily activities score (from the symptom with the highest interference score, ranging 1-4, with 4 indicating the highest burden on usual daily activities) for multimorbidity, compared with the sum of the interference score from two people with the two morbidity domains individually. $\mathrm{n}$ is the number of people with the multimorbidity combination. ENDO, endocrine; GASTRO, gastrointestinal; NEURO, neurological.

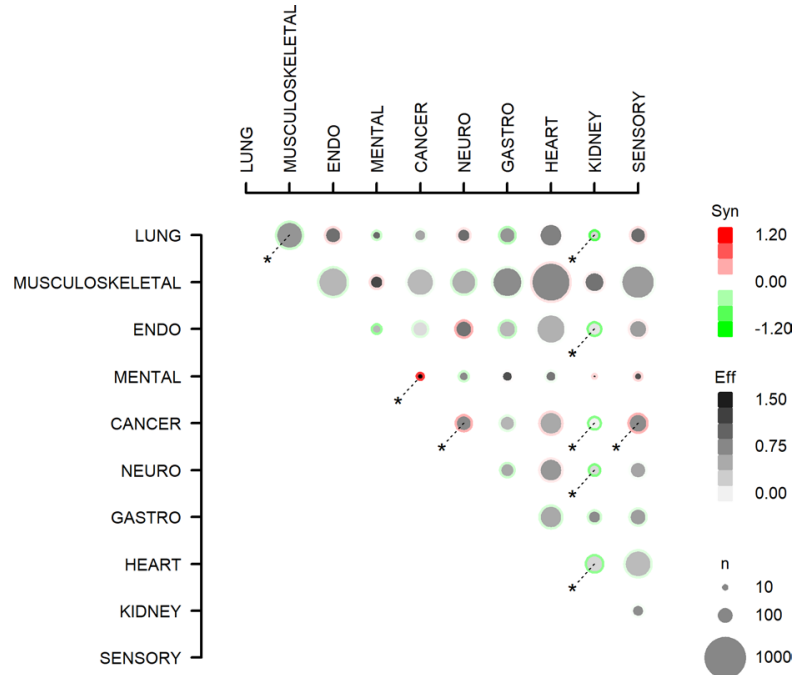

Figure 4 All (45) combinations of multimorbidity (two diagnosis groups) and the association with concern about symptoms. Effects with $\mathrm{p}<0.05$ are marked with *. Eff, effect: the concern score for the multimorbidity combination; Syn, synergy: the excess concern score (from the symptom with the highest concern score, ranging 1-4, with 4 indicating most concern) for multimorbidity, compared with the sum of the concern score from two people with the two morbidity domains individually. $\mathrm{n}$ is the number of people with the multimorbidity combination. ENDO, endocrine; GASTRO, gastrointestinal; NEURO, neurological.

burden in patients with multimorbidity was equivalent to the sum of the symptom burdens attributable to the individual morbidities. However, there were some exceptions to the overall additive pattern, most notably the combination sensory-cancer, where the symptom burden was supra-additive in all three components of symptom burden, and the combinations cancer-kidney and heartkidney, where all three components of symptom burden were infra-additive. Thus, sensory-cancer seemed to be especially burdensome, whereas cancer-kidney and heart-kidney seemed to be less burdensome than other combinations of multimorbidity.

\section{Comparisons with existing literature}

In this study, many participants reported symptoms independent of morbidities. Since symptoms are a main source of information when a doctor establishes a diagnosis ${ }^{9} 10$ and symptoms are important mediators for healthcare-seeking behaviour, ${ }^{28}$ the high number of symptoms independent of morbidity could seem unexpected. However, it was earlier shown that only a limited part of experienced symptoms end up being presented to a doctor. ${ }^{23}$ Furthermore, it highlights the fact that symptoms to some degree are prerequisites of human condition $^{7}$ in contrast to diagnoses that are created by medicine. ${ }^{10}$ However, symptoms have been shown to be frequent in the population, ${ }^{28} 29$ and when interviewed a large number of people reported experiencing symptoms within a 2-week period. ${ }^{30}$ Furthermore, it is reasonable to believe that symptom burden affects self-rated 
health, ${ }^{31} 32$ even though evidence suggests that it is not the symptom itself, but rather limitations on daily activities, worry and treatment burden related to the symptom that affect patients most. ${ }^{33}$ A person's self-rated health decreases with the number of chronic diseases they are living with, and this is most pronounced in younger and previously healthy people, ${ }^{34}$ which is in line with our results.

In this study, we found that the majority of multimorbidity combinations were additive and even had a levelling off effect. This could indicate that the definition of multimorbidity we used is clinically relevant, since the number of symptoms increases with the number of morbidities. While prognosis in multimorbidity is worse than could be predicted by adding the prognoses of individual diseases ${ }^{35}$ this is not the case for symptoms, according to the present study. This finding may, however, be explained by the overlap of symptoms between diagnoses. Another explanation could be that diagnoses are more likely to be made when patients have many contacts with the healthcare system. ${ }^{12}$ This could particularly add to the amount of diagnoses made without symptoms. Furthermore, it is known that older people seem to adapt to chronic conditions with a lower influence on their selfrated health as a result. ${ }^{35}$

This study shows that a mean of one new symptom is added for each morbidity, indicating that the symptom burden of patients with multimorbidity may be substantial. This may help clinicians to understand why patients with multimorbidity struggle to recognise which symptoms to focus on ${ }^{36}$ and also help us understand why patients with multimorbidity are sometimes overwhelmed by their symptom burden. ${ }^{513}$ With the number of morbidities they have to live with, multimorbidity can quickly become a complex issue for patients, ${ }^{37}$ not only because of the burden of symptoms, but also because of the burden of treatment, ${ }^{38}$ the organisational challenges ${ }^{38}$ and the demands from everyday life. ${ }^{33}$

In most combinations of multimorbidity, symptom burden was additive rather than supra-additive. However, there were a few exceptions; for example, the multimorbidity combination sensory-cancer was supra-additive. Patients with multimorbidity combinations that included cancer reported a supra-additive symptom burden, especially regarding their worry score. Cancer has a certain status in the general population as something fatal and cancer alarm symptoms can act as a mediator for making contact with a doctor. ${ }^{31}$

Participants with multimorbidity that included a kidney morbidity most often reported an infra-additive symptom burden, especially regarding their worry score. This could be explained by the fact that a kidney diagnosis is more likely to come from a laboratory test and general and often reported symptoms such as tiredness. Furthermore, in the kidney domain of our definition of multimorbidity, incontinence was an important and frequent diagnosis, but not necessarily a worrying one. ${ }^{39} 40$

\section{Strengths and limitations}

The population-based cohort and high response rate are strengths of our study, ${ }^{41}$ as well as the questionnaire containing a breadth of symptoms indicative of both serious and less harmful diseases, and the adjustments for several important factors. The relatively low prevalence of multimorbidity in this study compared with other studies can be explained by the definition based on 10 groups of diagnoses from secondary care. ${ }^{2}{ }^{42}$ The nationwide registers have high validity and they require referral to secondary care, thus ensuring that the resulting diagnoses have a certain seriousness. ${ }^{17}{ }^{18}$ In Denmark, primary care data on diagnoses are not available. However, we believe our broader definition, in contrast to simple disease counts, ${ }^{27} 43$ better grasps the burden and complexity of multimorbidity.

The selection of symptoms in the questionnaire is not exhaustive and are not selected in order to represent the morbidity domains; however, they may show preference towards certain domains; for example, there are many urinary tract-related symptoms relative to only a few kidney diagnoses. This may induce artificial differences between diagnoses groups. However, this does not bias the synergy estimates. Furthermore, the presence of diagnoses from secondary care contacts does not allow us to obtain adequate data on diagnosis duration, which could be of importance for symptom experience. ${ }^{44}$ However, the time limit of 4 weeks in the questionnaire was used to focus on relevant symptoms, while they can still be recalled..$^{15}$ Finally, we cannot completely rule out that the multimorbidity combinations being supra-additive or infra-additive may be a result of multiple testing.

\section{Implications}

It is well known that patients with multimorbidity face challenges in relation to their symptoms, ${ }^{5}$ and this study shows that symptom burden is, or rapidly becomes, substantial for these patients. Little is known about optimal management of symptoms, ${ }^{14}$ which underlines the need for increased attention to symptom burden among patients with multimorbidity. Initiatives such as patient diaries, describing the quality of disease management, paying attention to how patients explain their illness in order to understand their stories and life themes, ${ }^{45}$ and patient involvement in deciding the agenda and achieving realistic goals ${ }^{33}$ are probably valuable for optimal management. In conclusion, patients with multimorbidity add approximately one extra symptom for each additional morbidity, and symptom burden in these patients is additive, that is, equivalent to the sum of symptoms attributable to the individual morbidity.

Acknowledgements The authors are deeply grateful to Niels de Fine Olivarius for his dedicated support in this work.

Contributors TGW designed, wrote and edited the manuscript, researched the data, and contributed to the discussion. VS and DRN performed the analyses, researched the data, contributed to the discussion and reviewed/edited the manuscript. RK-R researched the data, contributed to the discussion and reviewed/ edited the manuscript. ADG, DJ and SR made important contributions to the 
discussion and content and reviewed/edited the manuscript. All authors approved the final version of the manuscript. TGW, VS and DRN have full access to data and take full responsibility for the truthfulness in the data and in the data analyses. TGW is the guarantor of this work.

Funding The study received funding from The Health Foundation (Danish) (grant number 19-B-0173).

Disclaimer The study sponsors had no role in study design, data collection, and analysis or in writing the manuscript.

Competing interests None declared.

Patient consent for publication Not required.

Ethics approval The Danish Symptom Cohort study was approved by the Ethics Committee and the National Board of Health. The Danish Data Protection Agency approved the Danish Symptom Cohort (journal number 2011-41-6651) and approved linkage with other registers (journal number 2015-231-0149). For the present analyses, the Danish Data Protection Agency, Statens Serum Institut and Statistics Denmark approved the use of register data. Administrative register data were anonymised, and reapproval from the Ethics Committee was not needed, nor did we need additional informed consent from the participants.

Provenance and peer review Not commissioned; externally peer reviewed.

Data availability statement Data may be obtained from a third party and are not publicly available. Data are stored at Statistics Denmark. To share data, approvals are required from the Danish Data Protection Agency and The Danish Health Data Authority.

Supplemental material This content has been supplied by the author(s). It has not been vetted by BMJ Publishing Group Limited (BMJ) and may not have been peer-reviewed. Any opinions or recommendations discussed are solely those of the author(s) and are not endorsed by BMJ. BMJ disclaims all liability and responsibility arising from any reliance placed on the content. Where the content includes any translated material, BMJ does not warrant the accuracy and reliability of the translations (including but not limited to local regulations, clinical guidelines, terminology, drug names and drug dosages), and is not responsible for any error and/or omissions arising from translation and adaptation or otherwise.

Open access This is an open access article distributed in accordance with the Creative Commons Attribution Non Commercial (CC BY-NC 4.0) license, which permits others to distribute, remix, adapt, build upon this work non-commercially, and license their derivative works on different terms, provided the original work is properly cited, appropriate credit is given, any changes made indicated, and the use is non-commercial. See: http://creativecommons.org/licenses/by-nc/4.0/.

\section{ORCID iDs}

Tora Grauers Willadsen http://orcid.org/0000-0001-7240-1944

Dorte Jarbol http://orcid.org/0000-0002-3241-4190

Susanne Reventlow http://orcid.org/0000-0002-4990-142X

\section{REFERENCES}

1 van den Akker MBF, Knottnerus JA. Comorbidity or multimorbidity: what's in a name? A review of literature. Eur $J$ Gen Pract 1996;2:65-70.

2 Barnett K, Mercer SW, Norbury M, et al. Epidemiology of multimorbidity and implications for health care, research, and medical education: a cross-sectional study. Lancet 2012;380:37-43.

3 Fortin M, Lapointe L, Hudon C, et al. Multimorbidity and quality of life in primary care: a systematic review. Health Qual Life Outcomes 2004;2:51.

4 Willadsen TG, Siersma V, Nicolaisdóttir DR, et al. Multimorbidity and mortality: a 15-year longitudinal registry-based nationwide Danish population study. J Comorb 2018;8:2235042X18804063.

5 Petrillo LA, Ritchie CS. The challenges of symptom management for patients with multimorbidity in research and practice: a thematic review. Prog Palliat Care 2016;24:262-7.

6 Nützel A, Dahlhaus A, Fuchs A, et al. Self-Rated health in multimorbid older general practice patients: a cross-sectional study in Germany. BMC Fam Pract 2014;15:1.

7 Hay MC. Reading sensations: understanding the process of distinguishing 'fine' from 'sick'. Transcult Psychiatry 2008;45:198-229.

8 Macleod U, Mitchell ED, Burgess C, et al. Risk factors for delayed presentation and referral of symptomatic cancer: evidence for common cancers. Br J Cancer 2009;101 Suppl 2:S92-101.
9 Malterud K, Guassora AD, Graungaard AH, et al. Understanding medical symptoms: a conceptual review and analysis. Theor Med Bioeth 2015;36:411-24.

10 Peterson MC, Holbrook JH, Von Hales D, et al. Contributions of the history, physical examination, and laboratory investigation in making medical diagnoses. West J Med 1992;156:163-5.

11 Undeland M, Malterud K. The fibromyalgia diagnosis: hardly helpful for the patients? A qualitative focus group study. Scand J Prim Health Care 2007;25:250-5.

12 Berkson J. Limitations of the application of fourfold table analysis to hospital data. Int J Epidemiol 2014;43:511-5.

13 Eckerblad J, Theander K, Ekdahl AW, et al. Symptom trajectory and symptom burden in older people with multimorbidity, secondary outcome from the RCT AGe-FIT study. J Adv Nurs 2016;72:2773-83.

14 Ritchie CS. Symptom burden: in need of more attention and more evidence. JAMA Intern Med 2013;173:1541-2.

15 Rasmussen S, Søndergaard J, Larsen PV, et al. The Danish Symptom Cohort: Questionnaire and Feasibility in the Nationwide Study on Symptom Experience and Healthcare-Seeking among 100000 Individuals. Int J Family Med 2014;2014:187280.

16 Pedersen CB. The Danish civil registration system. Scand J Public Health 2011;39:22-5.

17 Lynge E, Sandegaard JL, Rebolj M. The Danish national patient register. Scand J Public Health 2011;39:30-3.

18 Gjerstorff ML. The Danish cancer registry. Scand J Public Health 2011;39:42-5.

19 Mors O, Perto GP, Mortensen PB. The Danish psychiatric central research register. Scand J Public Health 2011;39:54-7.

20 Jensen VM, Rasmussen AW. Danish education registers. Scand $J$ Public Health 2011;39:91-4.

21 Petersson F, Baadsgaard M, Thygesen LC. Danish registers on personal labour market affiliation. Scand J Public Health 2011;39:95-8.

22 Baadsgaard M, Quitzau J. Danish registers on personal income and transfer payments. Scand J Public Health 2011;39:103-5.

23 Elnegaard S, Andersen RS, Pedersen AF, et al. Self-reported symptoms and healthcare seeking in the general population-exploring "The Symptom Iceberg". BMC Public Health 2015;15:685.

24 Rasmussen S, Larsen PV, Svendsen RP, et al. Alarm symptoms of upper gastrointestinal cancer and contact to general practice--A population-based study. Scand J Gastroenterol 2015;50:1268-75.

25 Jarbøl DE, Rasmussen S, Svendsen RP, et al. Barriers to contacting general practice with alarm symptoms of colorectal cancer: a population-based study. Fam Pract 2018;35:399-405.

26 Elnegaard S, Pedersen AF, Sand Andersen R, et al. What triggers healthcare-seeking behaviour when experiencing a symptom? results from a population-based survey. BJGP Open 2017;1:bjgpopen17X100761.

27 Willadsen TG, Bebe A, Køster-Rasmussen R, et al. The role of diseases, risk factors and symptoms in the definition of multimorbidity - a systematic review. Scand J Prim Health Care 2016;34:112-21.

28 Elliott AM, McAteer A, Hannaford PC. Revisiting the symptom iceberg in today's primary care: results from a UK population survey. BMC Fam Pract 2011;12:16.

29 McAteer A, Elliott AM, Hannaford PC. Ascertaining the size of the symptom iceberg in a UK-wide community-based survey. $\mathrm{Br} \mathrm{J}$ Gen Pract 2011;61:e1-11.

30 Hollnagel H. 40-åriges helbred. en epidemiologisk undersøgelse AF 40-årige kvinder OG mænd I Københavns amt. (health of 40-year olds. An epidemiological study of 40-year-old women and men in Copenhagen County). doctoral dissertation. Copenhagen: University of Copenhagen, 1985.

31 Jylhä M. What is self-rated health and why does it predict mortality? towards a unified conceptual model. Soc Sci Med 2009;69:307-16.

32 Waller G, Thalén P, Janlert U, et al. A cross-sectional and semantic investigation of self-rated health in the Northern Sweden MONICAstudy. BMC Med Res Methodol 2012;12:154.

33 Ørtenblad L, Meillier L, Jønsson AR. Multi-morbidity: a patient perspective on Navigating the health care system and everyday life. Chronic IIIn 2018;14:1-12.

34 Idler EL, Benyamini Y. Self-Rated health and mortality: a review of twenty-seven community studies. J Health Soc Behav 1997;38:21-37.

35 Leinonen R, Heikkinen E, Jylhä M. Changes in health, functional performance and activity predict changes in self-rated health: a 10-year follow-up study in older people. Arch Gerontol Geriatr 2002;35:79-92.

36 Eckerblad J, Theander K, Ekdahl A, et al. Symptom burden in community-dwelling older people with multimorbidity: a crosssectional study. BMC Geriatr 2015;15:1. 
37 Shippee ND, Shah ND, May CR, et al. Cumulative complexity: a functional, patient-centered model of patient complexity can improve research and practice. J Clin Epidemiol 2012;65:1041-51.

38 Bower P, Macdonald W, Harkness E, et al. Multimorbidity, service organization and clinical decision making in primary care: a qualitative study. Fam Pract 2011;28:579-87.

39 Rubach A, Balasubramaniam K, Elnegaard S, et al. Barriers to health care seeking with bothersome lower urinary tract symptoms among men-a nationwide study. Fam Pract 2019;36:743-50.

40 Rubach A, Balasubramaniam K, Storsveen MM, et al. Healthcareseeking with bothersome lower urinary tract symptoms among men in the Danish population: the impact of lifestyle and socioeconomic status. Scand J Prim Health Care 2019;37:155-64.

41 Galea S, Tracy M. Participation rates in epidemiologic studies. Ann Epidemiol 2007;17:643-53.
42 Britt HC, Harrison CM, Miller GC, et al. Prevalence and patterns of multimorbidity in Australia. Med $J$ Aust 2008;189:72-7.

43 Diederichs C, Berger K, Bartels DB. The measurement of multiple chronic diseases--a systematic review on existing multimorbidity indices. J Gerontol A Biol Sci Med Sci 2011;66:301-11.

44 Eccles M, Ford GA, Duggan S, et al. Are postal questionnaire surveys of reported activity valid? an exploration using general practitioner management of hypertension in older people. $\mathrm{Br} J$ Gen Pract 1999;49:35-8.

45 Kleinman. The meaning of symptoms and disorders and the personal and social meanings of illness (Chapter 1 and 2). in: the illness narratives: suffering, healing and the human condition United States of America. New York: Basic Books, 1988: 3-55. 